\title{
New thermal study of polymerization and degradation kinetics of methylene diphenyl diisocyanate
}

\author{
José Eduardo Estevam da Silva ${ }^{1} \cdot$ Rafael Turra Alarcon ${ }^{1} \cdot$ Caroline Gaglieri $^{1} \cdot$ Aroldo Geraldo Magdalena $^{1}$ \\ Luiz Carlos da Silva-Filho ${ }^{1} \cdot$ Gilbert Bannach $^{1}$
}

Received: 8 November 2017 / Accepted: 14 March 2018/Published online: 23 March 2018

(C) Akadémiai Kiadó, Budapest, Hungary 2018

\begin{abstract}
This work investigates the thermal polymerization process of a methylene diphenyl diisocyanate (MDI) monomer as well as its thermal degradation following the ICTAC recommendations. MDI monomer is widely used as a synthetic resin in the production of MDF panels, as it provides compaction of the eucalyptus fibers by polymerization. Thermogravimetry/ derivative thermogravimetric-differential thermal analysis (TG/DTG-DTA), differential scanning calorimetry, and midinfrared spectroscopy were used in this study. The polymerization process (An) and degradation (Fn) process exhibited activation energy equal to 149.70 and $80.22 \mathrm{~kJ} \mathrm{~mol}^{-1}$, respectively. The combined the FTIR and kinetic information makes it possible to suggest the mechanism reaction, which is an inedited data in literature.
\end{abstract}

Keywords Polymerization · Degradation · Methylene diphenyl diisocyanate $\cdot$ Non-isothermal kinetics $\cdot$ Thermal studies

\section{Introduction}

The 4,4'-methylene diphenyl diisocyanate (MDI, Fig. 1) in the pure monomer form is a light brown color, odorless high viscosity liquid, which reacts with water to release carbon dioxide [1]. MDI is a key component for the synthesis of polyurethane (PU) and is widely used in the processing of other products such as elastomers, sealants, and foams [2, 3], as well as in medium density fiberboard (MDF) production. MDI is a formaldehyde-free adhesive (environmental friendly) and an excellent substitute for urea-formaldehyde (UF) resins in the manufacture of wood fiber panels, as it provides greater durability and water resistance, avoiding early deterioration of the MDF panel $[4,5]$.

The present article is based on the lecture presented at SiAT VIII conference in Punta Grossa - Brazil on August 13-15, 2017.

Electronic supplementary material The online version of this article (https://doi.org/10.1007/s10973-018-7211-1) contains supplementary material, which is available to authorized users.

\section{Gilbert Bannach}

gilbert@fc.unesp.br

1 Chemistry Department, School of Sciences, São Paulo State University (UNESP), Bauru, SP 17033-260, Brazil
Duratex is a large Brazilian industry that makes MDF panel; like other industries around the world, they changed from using urea-formaldehyde resin to MDI, and consequently the production uses $75.0 \%$ less resin in panel fabrication. However, the MDI monomer is still more expensive than the common UF resin, and some manufacture problems appeared, such as wrong polymerization temperature, which caused low panel resistance, monomer waste, and manufacture spoil. Hence, it is important to determine the best polymerization temperature and activation energy of this process.

Non-isothermal kinetics studies have become an excellent choice to explain the reaction mechanism of polymers [4-10]. This kinetic approach is very important, due to its possible correlation between the activation energy in the conversion degree of the reaction or the extent of the reaction (polymerization). In the literature, two papers reported the non-isothermal kinetics studies of MDI polymerization process and final polymer degradation process $[11,12]$; however, both did not follow the ICTAC Kinetics Committee [13]. Thus, the present paper followed these recommendations and obtained new kinetics data for both process (polymerization and degradation).

These studies used thermogravimetry/derivative thermogravimetric-differential thermal analysis (TG/DTGDTA), differential scanning calorimetry (DSC), and mid- 
<smiles>O=C=Nc1ccc(Cc2ccc(N=C=O)cc2)cc1</smiles>

Fig. 1 Chemical structure of 4,4'-methylene diphenyl diisocyanate

infrared spectroscopy (MIR). The data from the nonisothermal kinetics studies was applied in licensed Software (Netzsch kinetics Neo Trial). These techniques produced results that help to clarify the MDI polymerization process, suggesting the formation of carbodiimide groups in polymer chain. Furthermore, the studied MDI thermal degradation step was showed to be a complex kinetic process (multiple steps).

\section{Materials and methods}

\section{MDI monomer}

MDI monomer was purchased from the Huntsman Corporation (Rubinate ${ }^{\circledR} 1840$ ).

\section{Thermal analyses: thermogravimetry-differential thermal analysis (TG-DTA) and differential scanning calorimetry (DSC)}

The TG-DTA curves were obtained using Netzsch equipment, model STA $449 \mathrm{~F} 3$, in $70 \mu \mathrm{L} \alpha$-alumina open crucibles with samples of about $14 \mathrm{mg}$, heating rate of $10.0^{\circ} \mathrm{C} \min ^{-1}$ in a dry air atmosphere (close to industrial ambient conditions) at a flow rate of $50.0 \mathrm{~mL} \mathrm{~min}^{-1}$, and a temperature range of $30.0-800.0{ }^{\circ} \mathrm{C}$.

The DSC analyses were carried out on METTLER TOLEDO equipment, model DSC $1 \mathrm{Star}^{\mathrm{e}}$ System. The DSC curves were obtained using $40 \mu \mathrm{L}$ closed aluminum crucibles with perforated lids, samples of about $11.0 \mathrm{mg}$ of monomer and at a heating rate of $10.0{ }^{\circ} \mathrm{C} \mathrm{min}{ }^{-1}$ in dry air atmosphere and a $50.0 \mathrm{~mL} \mathrm{~min}{ }^{-1}$ flow rate. The heating procedures were performed from 25.0 to $440.0^{\circ} \mathrm{C}$.

\section{Kinetics parameters}

The polymerization and degradation of MDI kinetic study was done under non-isothermal conditions, following the ICTAC recommendations [13]. The same equipment and parameters used before were employed, using different heating rate $\left(5.0,15.0,20.0\right.$, and $\left.25.0{ }^{\circ} \mathrm{C} \mathrm{min}^{-1}\right)$ as a complement to previously used $\left(10.0^{\circ} \mathrm{C} \mathrm{min}^{-1)}\right.$. All the data were processed using the Netzsch kinetics Neo Trial software by Netzsch [14]. The software admits the principles described by Ref [15], which use residual sum of squares (RSS).

\section{Mid-infrared spectroscopy (MIR)}

The spectra in the infrared region were obtained using a Nicolet iS10 FTIR spectrometer with a resolution of $4 \mathrm{~cm}^{-1}$ in the region of $4000-600 \mathrm{~cm}^{-1}$, using a Fourier transform spectrophotometer and accessory for the attenuated total reflectance (ATR) with germanium crystal.

\section{Results and discussion}

\section{TG-DTA and DTG analyses}

The TG/DTG-DTA curves for the MDI monomer and polymer are shown in Fig. 2. It is observed in TG curve for MDI monomer (Fig. 2a) that monomer was thermal stable until $180.0^{\circ} \mathrm{C}$, and this temperature is higher than the temperatures found in literature: 120 and $150{ }^{\circ} \mathrm{C}$ $[11,12]$. This difference is attributed to different equipments and analyzes conditions. There were three steps of mass loss, the first one indicating the polymerization process (TG $\Delta m=35.62 \%$ and Calculed $\Delta m=35.16 \%$ ), which is suggested for $\mathrm{CO}_{2}$ liberation and possible forming of carbodiimides group $(\mathrm{N}=\mathrm{C}=\mathrm{N})$. The polymerization was confirmed by the qualitative analysis in the test tube. The other two steps are related to degradation and oxidation of MDI polymer with $\Delta m=16.95 \%$ and $\Delta m=48.83 \%$, respectively.

The TG curve for MDI polymer (Fig. 2b) shows that the polymerization step occurred due to residual monomers (16.47\%). The degradation steps were similar to previous curve with $\Delta m=16.88 \%$ and $\Delta m=67.11 \%$, and no residual mass was found. Table 1 contains the details of the volatilization and decomposing steps of all the MDI samples as well as the thermal events in DTA.

\section{Differential scanning calorimetry (DSC)}

The DSC curve of MDI (Fig. 3) showed a glass transition (red highlighted) with onset at $131.4{ }^{\circ} \mathrm{C}$ and midpoint by ASTM at $136.1^{\circ} \mathrm{C}$ [16]. Furthermore, two exothermic events were observed with peaks at 295.0 and $330.0{ }^{\circ} \mathrm{C}$, respectively $\left(\Delta H=76.83 \mathrm{~J} \mathrm{~g}^{-1}\right)$, which are related to polymerization process. The exothermic peak at $428.0{ }^{\circ} \mathrm{C}$ suggests oxidation and degradation of the sample.

\section{Related kinetics studies for mass loss of MDI}

The polymerization process breaks some covalent bond and forms new covalent bonds between molecules (monomers). On the other hand, the degradation process breaks the polymer and forms smaller molecules such as 
Fig. 2 TG-DTA/DTG curves for MDI monomer (a) and MDI Polymer (b)
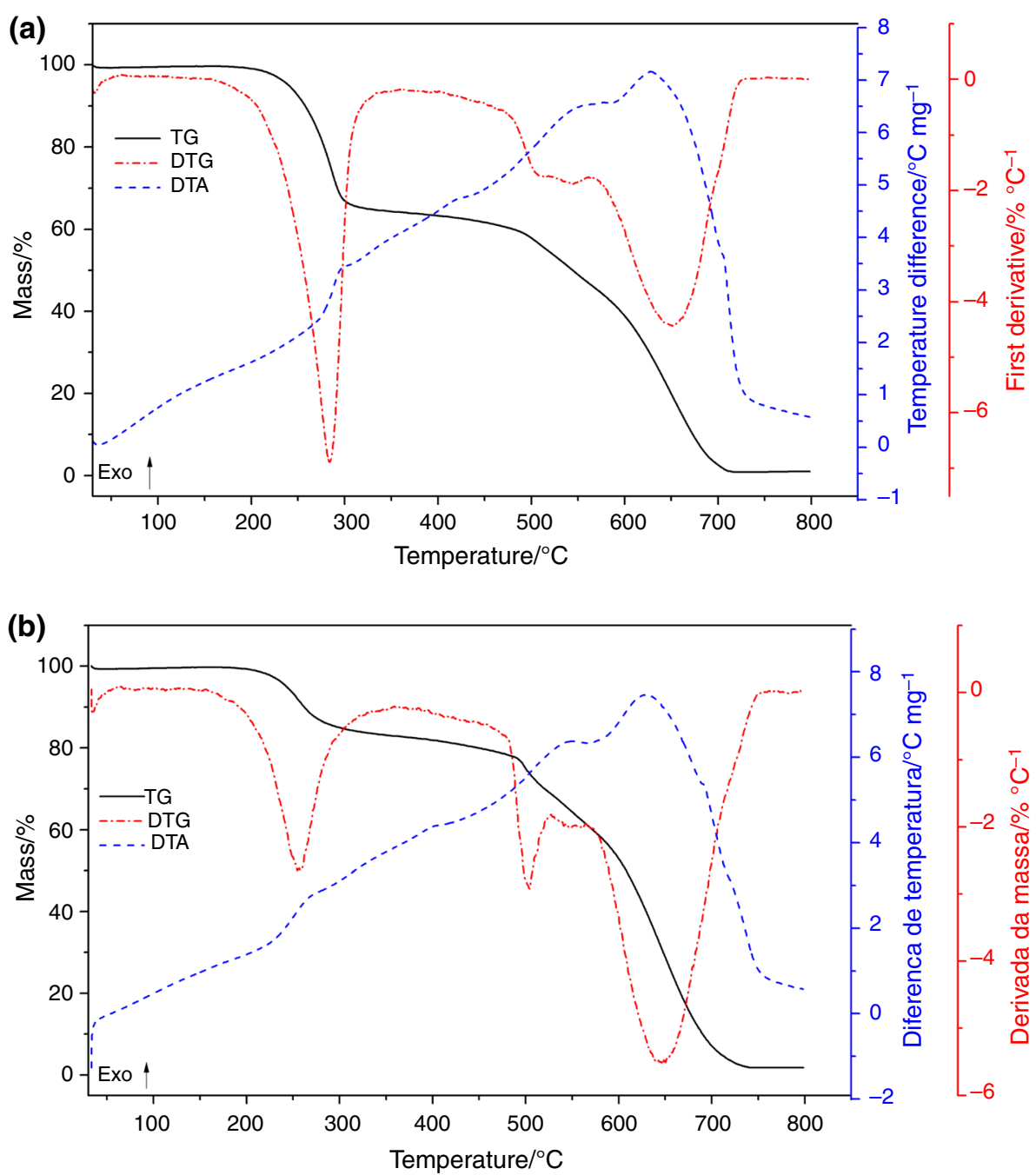

Table 1 Temperature $\left(\theta /{ }^{\circ} \mathrm{C}\right)$, mass loss $(\Delta m)$ and temperature peak $\left(T_{\mathrm{P}}\right)$ of the thermal events observed in each TG-DTA curve steps for each sample

\begin{tabular}{lllll}
\hline Sample & & 1st step & 2nd step & 3th step \\
\hline MDI monomer & $\theta /{ }^{\circ} \mathrm{C}$ & $180.0-347.2$ & $459.9-568.7$ & $568.7-739.4$ \\
& $\Delta m /$ & 35.62 & 15.55 & 44.75 \\
& $\%$ & & & \\
& $T_{\mathrm{P}} /{ }^{\circ} \mathrm{C}$ & $295.6 \uparrow$ & $459.9-579.8 *$ & $629.8 \uparrow$ \\
MDI polymer & $\theta /{ }^{\circ} \mathrm{C}$ & $180.0-330.0$ & $466.8-550.6$ & $550.6-761.0$ \\
& $\Delta m /$ & 16.01 & 16.88 & 65.01 \\
& $\%$ & & & \\
& $T_{\mathrm{P}} /{ }^{\circ} \mathrm{C}$ & $262.2 \uparrow$ & $540.5 \uparrow$ & $628.9 \uparrow$ \\
\hline
\end{tabular}

$\uparrow$ Exo up

*Exotherm

$\mathrm{CO}_{2}$ and $\mathrm{CO}$. The change of thermal conditions could result in various reactions in polymers, such as depolymerization and thermal degradation [4-10, 17, 18]. Furthermore, the polymerization and degradation processes usually occur at higher temperatures, which could initiate the conversion before reaching the isothermal temperature [13]. Thus, the use of isothermal conditions is inappropriate. Therefore, MDI polimerization and degradation processes were studied using the non-isothermal method.

There is already a study about the polymerization kinetic of MDI in literature [11]; however, the author did not follow the ICTAC recommendations. The model-free method used to determine the activation energy was the Ozawa-Flynn-Wall (OFW), which has low accuracy and is limited to linear heating rate conditions when compared with other methods. Moreover, this model should be used with iterative correction procedure for the value of activation energy [13]. The use of a computational software was not mentioned by the authors. Moreover, the authors:

- Used the linear regression, which should not be used for complex kinetics (this information will be discussed later) $[13,15]$;

- Did not show the $E_{\alpha}$ vs $\alpha$ dependency; 
Fig. 3 DSC curves for MDI

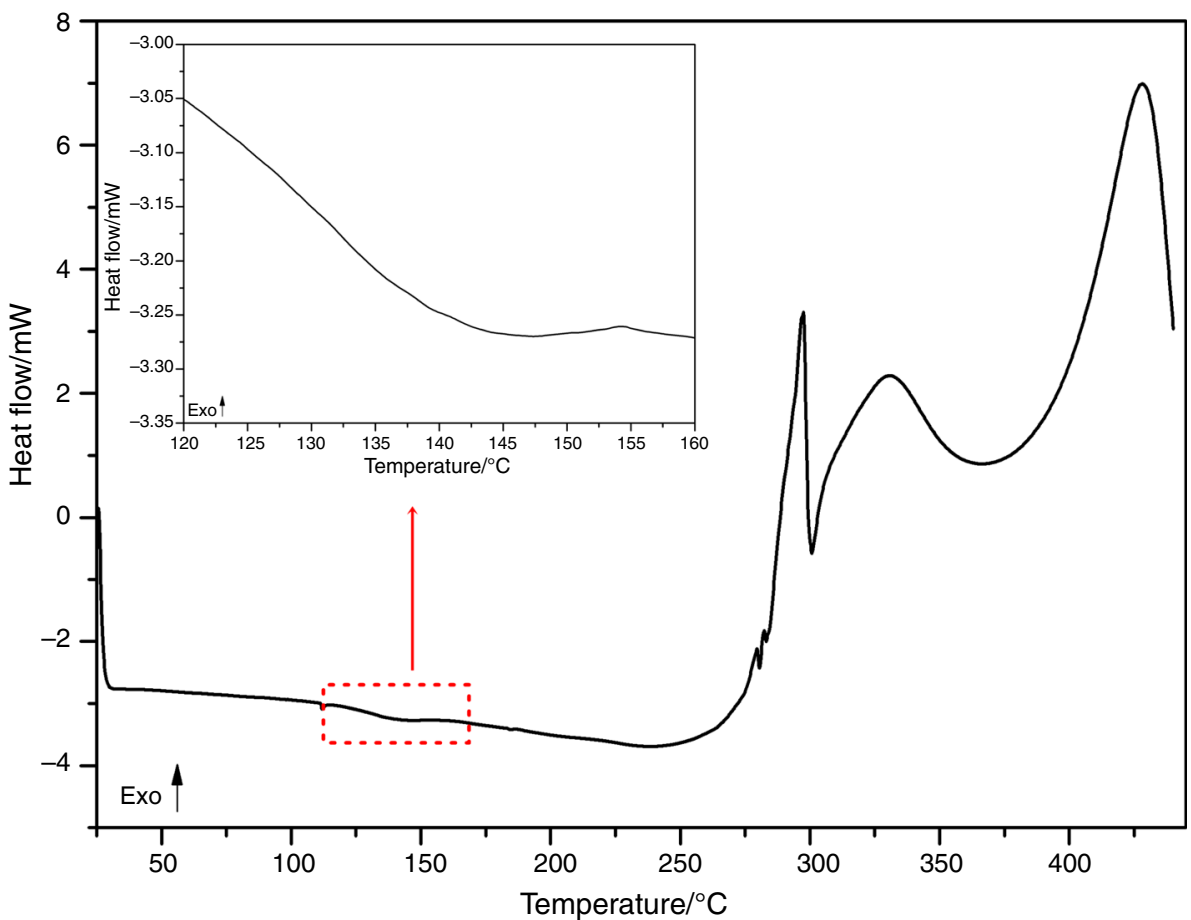

- Chose the better model (to determine the kind of reaction) comparing just the difference between the correlation coefficient $\left(R^{2}\right)$, which is not sufficient to make this choice $[13,15]$.

Keeping in mind this information, a study is necessary about the polymerization kinetic of MDI following the ICTAC recommendations. The TG curves in different heating rates are presented in Fig. 4.

All kinetic study is related to the reaction rate and is described by Eq. (1) [17, 18]:

$\frac{\mathrm{d} \alpha}{\mathrm{d} t}=k(T) f(\alpha)$

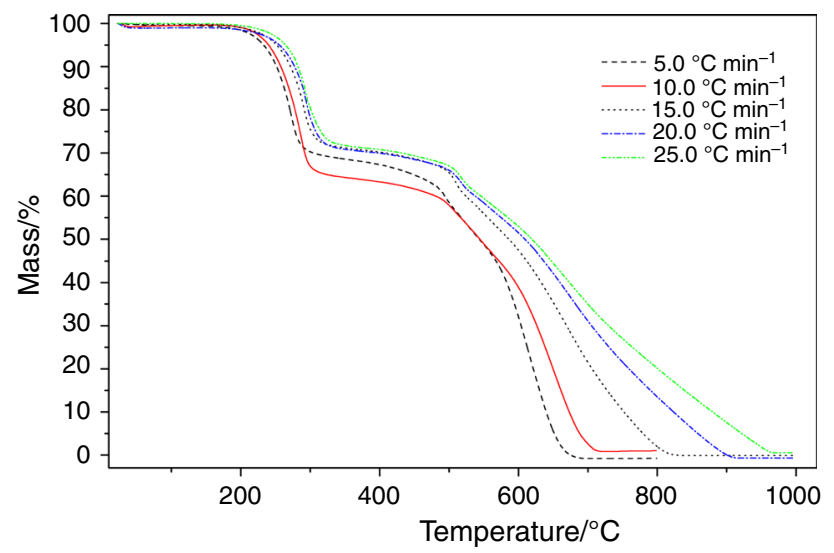

Fig. 4 TG curves at various heating rates for thermal decomposition of MDI under air. The heating rate of each experiment $\left(\mathrm{in} /{ }^{\circ} \mathrm{C} \min ^{-1}\right)$ is indicated in each curve where $\mathrm{d} \alpha / \mathrm{d} t$ is the reaction rate, $\alpha$ is the extent of the reaction, $k(T)$ is the rate constant, $t$ is time, $T$ is temperature, and $f(\alpha)$ is the reaction model. At constant extension of conversion, the reaction rate is only a function of temperature; therefore, it is possible to determine the activation energy for each extent of reaction without assuming any reaction model $[13,19]$. This information was important to determine if the polymeric and degradation process were kinetically complex. Using the expression proposed by Friedman, Eq. (2) [20], it was possible to obtain this information.

$\ln \left[\beta_{\mathrm{i}}\left(\frac{\mathrm{d} \alpha}{\mathrm{d} T}\right)_{\alpha, \mathrm{i}}\right]=\ln \left[f(\alpha) A_{\alpha}\right]-\frac{E_{\alpha}}{R T_{\alpha, \mathrm{i}}}$

In which $\beta_{\mathrm{t}}$ is associated to heating rate, parameters $A_{\alpha}$ and $E_{\alpha}$ are the pre-exponential factor and activation energy, respectively, and $R$ is the gas constant.

The extent of reaction rates was adjusted by Friedman method, Eq. (2), and the $E_{\alpha} v s \alpha$ graphic was plotted and is shown in Fig. 5. Note that the activation energy value oscillates between 188.8 (maximum value) and 131.9 (minimum value) $\mathrm{kJ} \mathrm{mol}^{-1}$ during the polymerization process, with some oscillations (shoulders) in the interval used ( 0.1 to 0.9$)$ his variation is typical of gelatinization process (oligomer formation) followed by crosslinking reaction (final polymer) and is attributed to mechanical change: from chemical to diffusion control $[13,21]$. It also indicates the occurrence of a complex process 


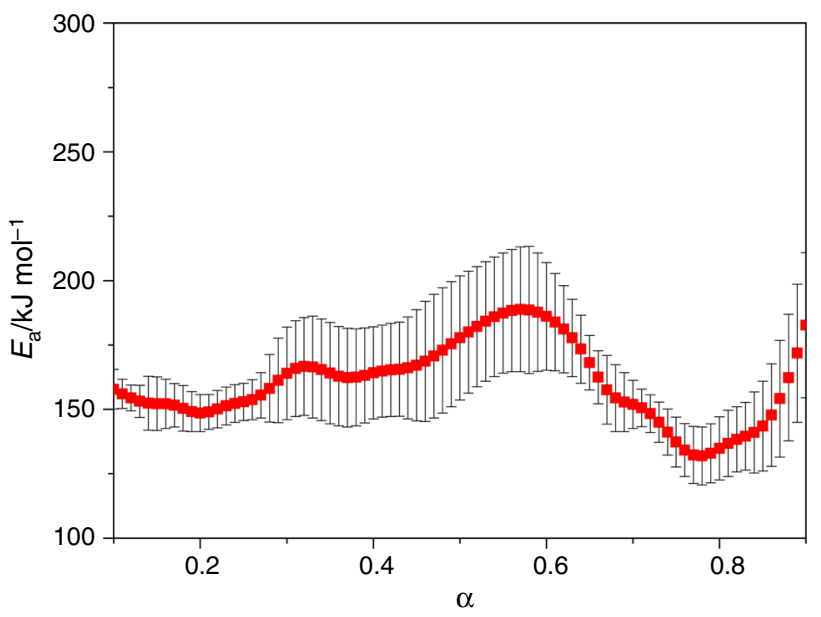

Fig. $5 E_{\alpha}$ dependence on $\alpha$ obtained by non-isothermal analyses of TG data for first mass loss of MDI polymer using Friedman method

$[7,13,17,18]$. The activation energy behavior suggests the formation of more than one intermediate reaction.

In similar situations, when the maximum and minimum values of activation energy oscillate more than $20-30 \%$ of the average, the use of computationally complex methods is recommended [13]. Using the option nonlinear modelfitting offered by the software Netzsch kinetics Neo Trial and considering an independent reaction, the best method obtained for polymerization process was $n$-dimensional nucleation (An), which found values of activation energy and pre-exponential factor equal to $149.7 \mathrm{~kJ} \mathrm{~mol}^{-1}$ and $1.3 \times 10^{12} \mathrm{~s}^{-1}$, respectively. The $E_{\alpha}$ value found by Ref [11] (50-60 $\mathrm{kJ} \mathrm{mol}^{-1}$ ) was three times lower than the value found in this work.

This is a diffusional method [4, 22] and is in agreement with the $E_{\alpha} v s \alpha$ dependence obtained by Friedman method. The Ref $[11,12]$ shows that just the coefficient correlation $\left(R^{2}\right)$ value is not enough to choose a better solution. The $F$ test was considered, which gives just one ideal solution, in this study (An) [15, 23]. All values of activation energy, logarithm of pre-exponential factor, correlation coefficient, and $F_{\text {exp }}$ are shown in Table S1.

Reference [12] showed the activation energy of MDI polymerization, and although less detailed than Ref [11], the average $E_{\alpha}$ value found was more consistent with the polymerization process $\left(200.99 \mathrm{~kJ} \mathrm{~mol}^{-1}\right)$. The author also found the activation energy of the MDI degradation process $\left(259.94 \mathrm{~kJ} \mathrm{~mol}^{-1}\right)$. In both cases, they used the OFW method, which has some disadvantages, as previously mentioned, and did not follow the ICTAC recommendations [13].

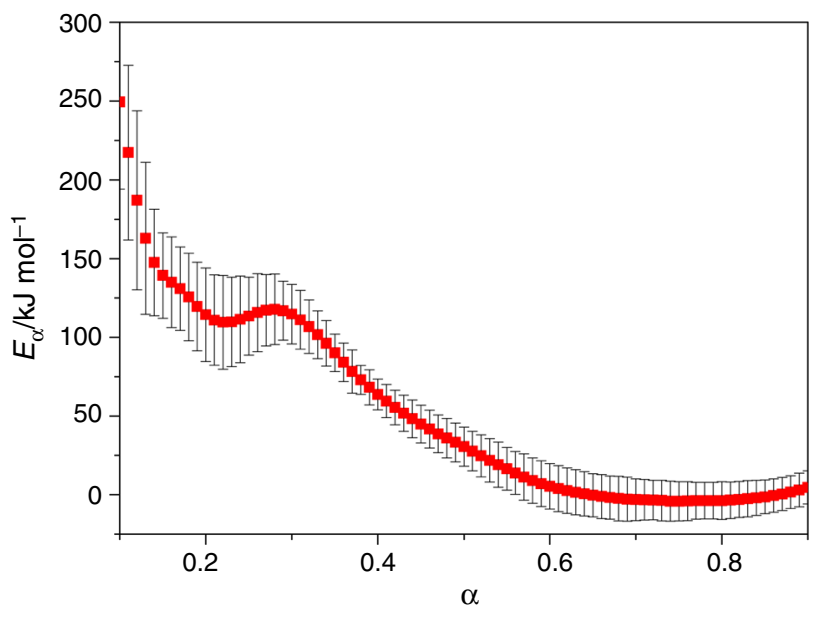

Fig. $6 E_{\alpha}$ dependence on $\alpha$ obtained by non-isothermal analyses of TG data for second mass loss of MDI polymer using Friedman method

The $E_{\alpha}$ vs $\alpha$ dependency obtained by the Friedman analysis for degradation of MDI polymer is shown in Fig. 6. The decrease of activation energy is noted (249.4 to $4.7 \mathrm{~kJ} \mathrm{~mol}^{-1}$ ), but with three different behaviors associated with this process. The observed polymerization process is related to the complex degradation process that generates several products, as mentioned by the Ref. [12]. The activation energy values varied more than 20-30\%; therefore, the nonlinear regression was used to determine the best model. Considering the $R^{2}(0.983147)$ and the $F_{\exp }(1.00)$ values, the best model obtained was the n-order reaction (Fn), which provided $E_{\alpha}=80.22 \mathrm{~mol}^{-1}, A=97.18 \mathrm{~s}^{-1}$.

The best model for degradation process exhibited $F_{\text {exp- }}$ $=1.00$. This information indicates that there is no significant difference between the experimental and the theoretical data provided by the best kinetic model. All values of activation energy, logarithm of pre-exponential factor, correlation coefficient, and $F_{\exp }$ obtained for MDI polymer degradation are shown in Table S2.

Analyzing both values of activation energy (polymerization and degradation), it is observed that the first value is higher than the degradation one. This could be explained due to the polymerization reaction of isocyanates requires high energy; thus, it is used catalysts or heating in these syntheses [24-26]. Another reason, is not possible to compare these, due to be different process and different materials (1st Step: monomer to polymer and 2nd step: polymer degradation).

The lifetime to achieve 5\% of each process (polymerization and degradation) was calculated as described by 

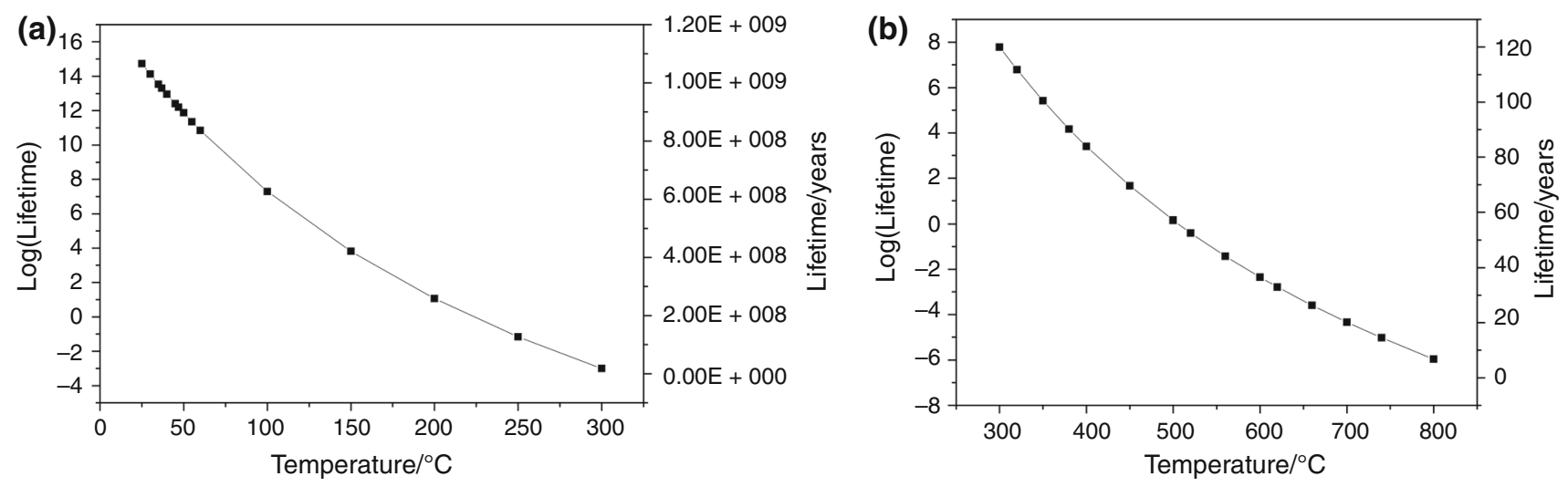

Fig. 7 Lifetime versus necessary temperature for MDI polymerization (a) and MDI degradation (b)

ASTM 1641 and 1877 [27, 28] following Eq. 3, with the resulting graph shown in Fig. 7a.

$\log t_{\mathrm{f}}=\frac{E_{\mathrm{a}}}{\left(2.303 R T_{\mathrm{f}}\right)+\log \left[\frac{E_{\mathrm{a}}}{(R \beta)}\right]-a}$

The lifetime to polymerize $5 \%$ at $200.0{ }^{\circ} \mathrm{C}$ of MDI is about $11 \mathrm{~min}$, and the lifetime of MDI polymer to degrade $5 \%$ at $500.0{ }^{\circ} \mathrm{C}$ is about $1 \mathrm{~min}$, with the resulting graph shown in Fig. 7b. However, these results are just a time

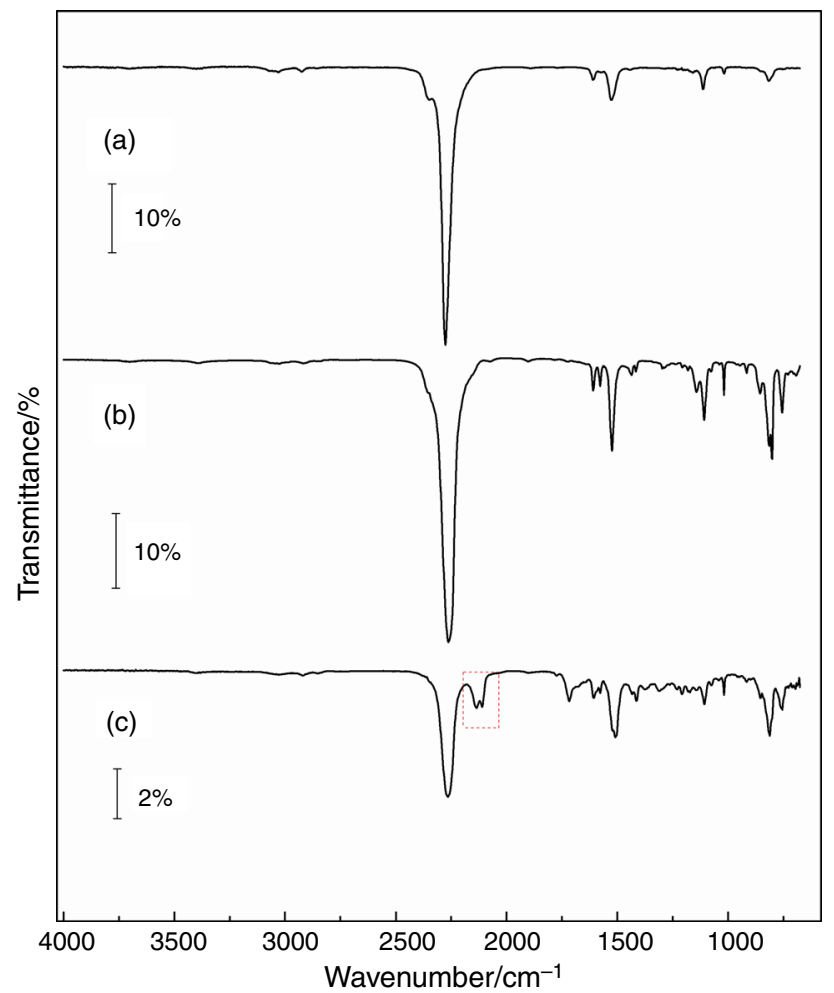

Fig. 8 IR spectrum of MDI literature (a), MDI monomer (b), and MDI polymer (c) estimate and can be used to compare different raw materials of same product (MDI) in the industry or determine the better temperature for work and storage.

\section{Mid-infrared spectroscopy}

The infrared absorption spectra for standard MDI [29] of MDI and MDI polymer are presented in Fig. 8. The MIR spectrum of standard MDI (Fig. 8a) shows an intense characteristic peak at $2280 \mathrm{~cm}^{-1}$ attributed to stretching of the isocyanate group $(\mathrm{R}-\mathrm{N}=\mathrm{C}=\mathrm{O})$ [30]. The MDI monomer had the same peak at $2266 \mathrm{~cm}^{-1}$ related to stretching of the isocyanate group. However, the MDI polymer presented a different spectrum, in that the respective isocyanate peak at $2266 \mathrm{~cm}^{-1}$ had less intensity and two new peaks appeared at 2110 and $2136 \mathrm{~cm}^{-1}$. These peaks are related to symmetrical and asymmetrical stretching of carbodiimide group ( $\mathrm{R}-\mathrm{N}=\mathrm{C}=\mathrm{N}-\mathrm{R}$ ), formed in the polymerization process $[30,31]$. The carbodiimide formation in this process is confirmed by ${ }^{15} \mathrm{~N}-\mathrm{NMR}$ in literature [32].

TG and FTIR data indicated a reaction mechanism for carbodiimide formation. Ref [12] reports this information; however, without any literature explaining the reaction mechanism. This paper suggested the pericyclic reaction, which results in a four-member ring (uretidinone). Then, a rearrangement occurs and forms a second intermediate: a carbamate four-member ring, which rearranges one more time releasing carbon dioxide gas and forming the final product, a carbodiimide polymer. This is a favorable reaction due to the gas formed as one of products [33]. The suggested mechanism reaction is shown in Fig. 9. This mechanism is in agreement with the activation energy behavior observed in Fig. 5. Some oscillations could be associated with these intermediate reactions. 


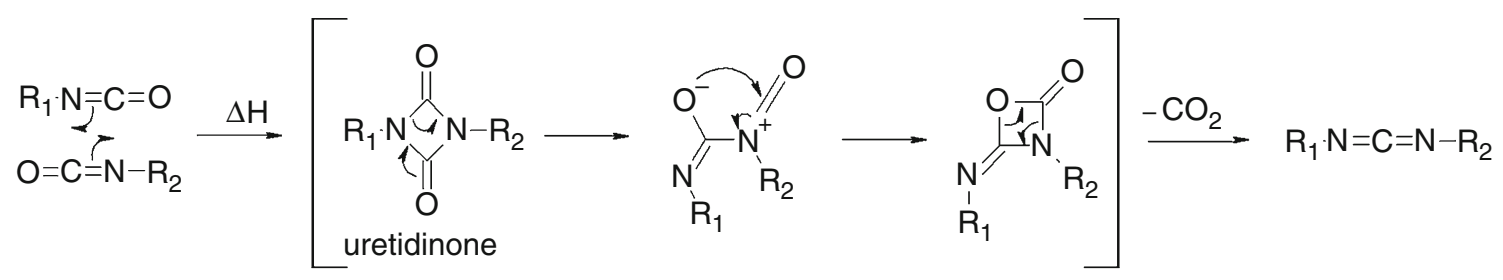

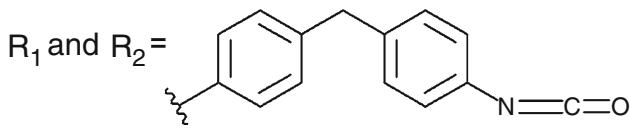

Fig. 9 Reaction mechanism for carbodiimide formation

\section{Conclusions}

The TG/DTG-DTA curves of MDI monomer and its polymer presented similar behavior of thermal stability at $180.0^{\circ} \mathrm{C}$ and three steps of mass loss. The DSC showed two exothermic peaks at 295.0 and $330.0^{\circ} \mathrm{C}$, associated to polymerization, followed by decomposition event at $428.0^{\circ} \mathrm{C}$. The MIR spectra confirmed the presence of carbodiimides in MDI polymer with characteristic peaks at 2110 and $2136 \mathrm{~cm}^{-1}$, and it was possible to suggest a mechanism for the carbodiimide formation. Furthermore, the polymerization and degradation processes were analyzed by kinetic studies following the ICTAC recommendations and then compared to literature results. The polymerization process exhibits activation energy equal to $149.7 \mathrm{~kJ} \mathrm{~mol}^{-1}$ and the best model obtained by nonlinear regression was the $A n$. In the degradation process, the activation energy decreased from 450.0 to $80.22 \mathrm{~kJ} \mathrm{~mol}^{-1}$ and the best model obtained was the $F n$. These data showed more accuracy than the previously literature data, due to the use of software and nonlinear regression. Finally, it was possible to calculate the lifetime as $5 \%$ of polymerization at $200{ }^{\circ} \mathrm{C}(11 \mathrm{~min})$ and degradation at $500.0^{\circ} \mathrm{C}(1 \mathrm{~min})$, this information could be used to determine the better temperature for work and storage or compare different raw materials of same product (MDI) in the industry.

Acknowledgements The authors wish to thank CAPES (proc. 024/2012 Pro-equipment), POSMAT/UNESP) and FAPESP (processes: 2013/09022-7 and 2017/08820-8), CNPq (Processes $302267 / 2015-8$ and 302753/2015-0) for financial support, as well as Netzsch-Brazil for providing kinetic computational program (Netzsch kinetics Neo Trial)

\section{References}

1. Product Safety Assessment. DOW Modified Methyl Diphenyl Diisocyanate (MDI) Products 2015;1-8.
2. Hagerman L, Law B, Bledsoe T, Hettick J, Kashon M, Lemons A, Wisnewski A, Siegel P. The influence of diisocyanate antigen preparation methodology on monoclonal and serum antibody recognition. J Occup Environ Hygiene. 2016;13:829-39.

3. Fug F, Rohe K, Vargas J, Nies C, Springborg M, Possart W. 4,4'methylene diphenyl diisocyanate-conformational space, normal vibrations and infrared spectra. Polymer. 2016;99:671-83.

4. Vyazovkin S, Sbirrazzuoli N. Isoconversional kinetic analysis of thermally. stimulated processes in polymers. Macromol Rapid Commun. 2006;27:1515-32.

5. Sbirrazzuoli N, Vincent L, Mija A, Guigo N. Integral, differential and advanced isoconversional methods Complex mechanisms and isothermal predicted conversion-time curves. Chemom Intell Lab Syst. 2009;96:219-26.

6. Vyazovkin S, Vincent L, Sbirrazzuoli N. Thermal denaturation of collagen analyzed by isoconversional method. Macromol Biosci. 2007;7:1181-6.

7. Jablonskli AE, Lang AJ, Vyazovkin S. Isoconversional kinetics of degradation of polyvinylpyrrolidone used as a matrix for ammonium nitrate stabilization. Thermochim Acta. 2008;474:78-80.

8. Peterson JD, Vyazovkin S, Wight CA. Kinetic study of stabilizing effect of oxygen on thermal degradation of poly(methylmethacrylate). J Phys Chem B. 1999;103:8087-92.

9. Arisawa H, Brill TB. Kinetics and mechanisms of flash pyrolysis of poly(methyl methacrylate) (PMMA). Combust Flame. 1997;109:415-26.

10. Arii T, Ichihara S, Nakagawa H, Fujii N. A kinetic study of the thermal decomposition of polyesters by controlled-rate thermogravimetry. Termochim Acta. 1998;319:139-49.

11. Zhang Q, Li H, Liu H. Study on polymerization kinetics of methylene diphenyl diisocyanate. Acta Chim Sinica. 2011;69:605-9.

12. Zhang J, Tang Y, Liu J, Chen Y. Thermal stability and thermal degradation reaction kinetics of 4,4'-Diphenylmethane diisocyanatetrimer. Asian J Chem. 2014;26:1527-9.

13. Vyadzovkin S, Burnham AK, Criado JM, Pérez-Maqueda LA, Popescu C, Sbirrazzuoli N. ICTAC Kinetics committee recommendations for performing kinetic computations on thermal analysis data. Thermochin Acta. 2011;520:1-19.

14. Netzsch-Thermokinetics. https://kinetics.netzsch.com/en/. Acessed 09 Sep 2017.

15. Opfermann J. Kinetic analysis using a multivariate nonlinear regression. J Therm Anal Calorim. 2000;60:641-58.

16. American Society for Testing and Materials-ASTM. ASTME1356: Standard test method for assignment of the glass transition temperatures by differential scanning calorimetry. West Conshohocken: ASTM; 2014. 
17. Flynn JH, Wall LA. A quick, direct method for the determination of activation energy from thermogravimetric data. Polym Lett. 1966;4:323-8.

18. Ozawa T. A new method of analyzing thermogravimetric data. Bull Chem Soc Jpn. 1965;38:1881-6.

19. Moukhina E. Determination of kinetic mechanisms for reactions measured with thermoanalytical instruments. J Therm Anal Calorim. 2012;109(1203-121):4.

20. Friedman HL. Kinetics of thermal degradation of char-forming plastics from thermogravimetry. Application to a phenolic plastic. J Polym Sci. 1964; 183-195.

21. Pielichowski K, Czub P, Pielichowski J. The kinetics of cure of epoxides and related sulphur compounds study by dynamic DSC. Polymer. 2000;41:4381-8.

22. Peterson JD, Vyadzovkin S, Wight CA. Kinetics of the thermal and thermo-oxidative degradation of polystyrene, polyethylene and poly(propylene). Macromol Chem Phys. 2001;202:775-84.

23. Miller JN, Miller JC. Statistics and chemometrics for analytical chemistry. 6th ed. Harlow: Pearson Education Limited; 2010.

24. Monagle JJ. Carbodiimides III: conversion of isocyanates to carbodiimides catalyst studies. J Organ Chem. 1962;27:3851-5.

25. Puszka A, Kultys A. New thermoplastic polyurethane elastomer based on aliphatic diisocyanate. J Therm Anal Calorim. 2017;128:407-16.
26. Kong W, Lei Y, Jiang Y, Lei J. Preparation and thermal performance of polyurethane/PEG as novel form-stable phase change materials for thermal energy storage. J Therm Anal Calorim. 2017;130:1011-9.

27. American Society for Testing and Materials-ASTM. ASTME1641: Standard test method for decomposition kinetics by thermogravimetry. West Conshohocken: ASTM; 1999.

28. American Society for Testing and Materials-ASTM. ASTME1877: Standard practice for calculating thermal endurance of materials from thermogravimetric decomposition data. West Conshohocken: ASTM; 1999.

29. National Institute of Standards and Technology-NIST. IR Spectrum methylene diphenyl diisocyanate. Webbook NIST; 2009.

30. Silverstein RM, Webster FX, Kiemle DJ, editors. Spectrometric Identification of organic compounds. 7th ed. Wiley, 2005.

31. Hatchett DW, Kodipilli G, Kinianjui JM, Benincasa F, Sapochak L. FTIR Analysis of thermally processed PU foam. Polym Degrad Stab. 2005;87:555-61.

32. Duff DW, Maciel GE. Monitoring the thermal degradation of a isocyanurate-rich MDI-based resin by ${ }^{15} \mathrm{~N}$ and ${ }^{13} \mathrm{C} \mathrm{CP} / \mathrm{MAS}$ NMR. Macromol. 1991;24:651-8.

33. Brown TE, LeMay HE, Bursten BE. Murphy C, Woodward P.Chemistry: the central science. 9th ed. Boston: Pearson, 2005. 